\title{
Long noncoding RNA AC096655.1-002 has been officially named as gastric cancer-associated transcript 1, GACAT1
}

\author{
Bingxiu Xiao • Junming Guo
}

Received: 17 May 2013 / Accepted: 3 June 2013 / Published online: 12 June 2013

(C) International Society of Oncology and BioMarkers (ISOBM) 2013

Shortly after the article entitled "Decreased expression of long noncoding RNA AC096655.1-002 in gastric cancer and its clinical significance" published in Tumor Biology [1], the long noncoding RNA AC096655.1-002 that studied in this paper has been officially named by HUGO Gene Nomenclature Committee (HGNC). Readers can find the detail information from Human Gene Nomenclature Database (www.genenames.org/hgnc-searches). Its HGNC ID is HGNC: 48336. Its synonym is AC096655.1-002, LINC00876, "long intergenic non-protein coding RNA 876." Its gene is located at $2 \mathrm{q} 12.3$.

As long noncoding RNA AC096655.1-002 was first found associated with gastric cancer, the committee has named it as "gastric cancer-associated transcript 1, GACAT1." That is, approved symbol is GACAT1 and approved name is gastric cancer-associated transcript 1 (http://www.genenames.org/data/ hgnc_data.php?hgne_id=48336). GACAT1 has been found significantly downregulated in gastric cancer tissues compared with paired-adjacent non-tumorous tissues. Its expression level was significantly correlated with lymph node metastasis, distant metastasis, TNM stages, and differentiation [1]. These results indicated that GACAT1 may be a potential biomarker in the diagnosis of gastric carcinoma.

Conflicts of interest None

\section{Reference}

1. Sun W, Wu Y, Yu X, Liu Y, Song H, Xia T, et al. Decreased expression of long noncoding RNA AC096655.1-002 in gastric cancer and its clinical significance. Tumor Biol. 2013. doi:10.1007/s13277-0130821-0.

B. Xiao $\cdot$ J. Guo $(\bowtie)$

Zhejiang Provincial Key Laboratory of Pathophysiology,

Ningbo University School of Medicine, Ningbo 315211, China

e-mail: junmingguo@yahoo.com 